\title{
Religion and Prejudice Across Cultures: A Test of the Threat-Constraint Model
}

\author{
Jasper Van Assche ${ }^{1}$ Joaquín Bahamondes $^{2}$, \& Chris Sibley $^{2}$ \\ ${ }^{1}$ Department of Developmental, Personality and Social Psychology, Ghent University \\ ${ }^{2}$ School of Psychology, University of Auckland
}

Paper in press in Social Psychological \& Personality Science

Corresponding author: Jasper Van Assche

Department of Developmental, Personality and Social Psychology

Faculty of Psychology and Educational Sciences, Ghent University

Henri Dunantlaan 2, B-9000 Ghent (Belgium)

E-mail: Jasper.VanAssche@UGent.be

Telephone: +3292646424

Fax: +3292646499

ORCID: 0000-0002-2570-2928

Author note: The authors declare that there are no potential conflicts of interest with respect to the research, authorship, and/or publication of this article.

Word count: 4986

Running head: Worldwide religion-prejudice associations 


\begin{abstract}
The present set of studies investigated the role of being religious in the prediction of various forms of prejudice. Following the Threat-Constraint Model, we predicted that contexts characterized by high threat attenuate —or constrain — the relationship between individual differences in being religious on the one hand, and anti-gay prejudice and sexism on the other. A worldwide investigation of these regional constraints was conducted in the Americas Barometer (125,984 individuals nested in 20 countries; Study 1), the World Value Survey (69,798 individuals nested in 45 countries; Study 2), and the European Social Survey (44,386 individuals nested in 274 NUTS-regions; Study 3). Results identify a key moderating role of Hofstede's cultural dimensions, revealing strong associations between religion and prejudice in regions low in power distance, uncertainty avoidance, masculinity and collectivism, whereas the religion-prejudice association is constrained (i.e., weaker and often absent) in regions high on those cultural dimensions.
\end{abstract}

Key words: Religion; Hofstede; culture; anti-gay prejudice; sexist attitudes 
Allport (1966) claimed that "the woof of bigotry" is woven into the fabric of all religion. Put differently, being religious is a strong predictor of being bigoted. Numerous studies have shown robust, positive associations between religiosity on the one hand; and racism (e.g., Hall, Matz, \& Wood, 2010), sexism (e.g., Van Assche, Koç, \& Roets, 2019), and anti-gay prejudice (e.g., Whitley, 2009), on the other. Accordingly, Glick, Lameiras, and Castro (2002) asserted that researchers should consider the role religion plays in people's lives when investigating gender- and sexual orientation-related attitudes.

The association between religion and prejudice is also likely to vary across countries. According to the Threat-Constraint Model (TCM; Sibley, Osborne, \& Duckitt, 2012), associations between individual dispositions and prejudice are attenuated in contexts characterized by greater situational threat. Studies applying this person $\times$ context interaction perspective consistently corroborate that individual differences are most predictive of attitudes when situational factors (high levels of threat, De keersmaecker et al., 2017; or extreme social norms, Van Assche, Roets, De keersmaecker, \& Van Hiel, 2017) do not inhibit individual tendencies. Under extreme conditions, however, the influence of individual differences is diminished.

\section{How homophobia and sexism are predicted by the person and the context}

Two recent large-scale studies provide robust evidence for the TCM with regards to anti-gay bias. Navarro, Barrientos, Gómez, and Bahamondes (2019) showed that religiosity decreased tolerance of homosexuality only in post-materialist Latin American countries, whereas this effect was not significant in countries low in post-materialism (see also Adamczyck \& Pitt, 2009). Similarly, Hoffarth, Hodson, and Molnar (2018) reported strong negative effects of religious attendance on gay-adoption support, tolerance of homosexuality, and accepting homosexual neighbors in countries high in gay rights recognition. These 
relationships were absent in countries low in gay rights recognition. As such, the role of religiosity in individual differences in anti-gay bias seems constrained in contexts that can be defined as highly threatening. To date, individual differences in sexism have not yet been examined as a function of individual-level religion and context-level variables, but we predict similar stronger associations in low-threatening contexts, versus weaker associations in highthreatening contexts. We use Hofstede's cultural dimensions as higher-level moderators in this study.

Hofstede (1980) argued that important between-country cultural differences are captured by four dimensions: power distance (i.e., the cultural acceptance of hierarchical relations and unequal distribution), uncertainty avoidance (i.e., the intolerance for ambiguity and emphasis on strict rules within a culture), masculinity-femininity (i.e., the adherence to traditionally masculine societal values such as achievement and success), and individualismcollectivism (i.e., the emphasis on individual freedom of action vs. group cohesion). These dimensions should shape how individuals within that culture feel towards outgroups. For instance, power distance, uncertainty avoidance, masculinity, and collectivism should foster anti-immigrant sentiment (Leong \& Ward, 2006). Power distance, specifically, enhances sexism (Glick, 2006), whereas ethnic prejudice is more likely in collectivistic cultures (Triandis, 1995). Hence, we hypothesize that anti-gay prejudice and sexism will be higher in countries high on the aforementioned cultural dimensions.

Furthermore, what unifies these dimensions, is that they all reflect a type of threat as operationalized by TCM. For instance, countries high in power distance often adhere to capitalist ideology, have autocratic governments with frequent corruption, and are often characterized by greater average levels of competition and income inequality (see Onraet, Van Hiel, \& Cornelis, 2013). Moreover, countries high in uncertainty avoidance and collectivism show greater mean levels of respect for authorities, conservatism, and intolerance, and highly 
masculine countries are often the ones lower in democratic and post-materialist values (Onraet et al., 2013; Welzel \& Inglehart, 2016). In sum, cultures high in power distance, uncertainty avoidance, masculinity, and collectivism, can be considered high-threatening contexts, constraining the link between religion and anti-gay or sexist attitudes.

\section{Study 1}

\section{Method}

\section{Participants}

Data for the subsequent empirical analysis are drawn from the Americas Barometer (2004-2014), a comparative survey administered by the Latin American Public Opinion Project, covering 28 North-, Central-, and South-American, and Caribbean nations. For 8 countries (Bahamas, Barbados, Belize, Bolivia, Guyana, Haiti, Nicaragua, and Paraguay), scores on the cultural dimensions were unavailable, these were omitted from analyses. All other countries and their cultural dimension scores are presented in Table A in the Supplemental Online Materials (SOM). The final sample consisted of 125,984 individuals from 20 countries $\left(M_{\text {age }}=40.04, S D_{a g e}=16.19\right.$, with 51.1\% women; $M=6,299$ observations per country; more sample information in SOM).

\section{Measures}

Religious affiliation. Ten percent of the sample had no religious affiliation, $62.0 \%$ identified as Catholic, 23.5\% identified as Protestant, and 4.3\% adhered to an 'other, nonChristian' religion. We dummy-coded this variable with 0 ("No religion") and 1 ("A religion"). 
Cultural dimensions. Country scores on the four cultural dimensions were retrieved from https://www.hofstede-insights.com (see Table B in SOM for means, standard deviations, and correlations between the dimensions).

Anti-gay prejudice. Two items tapped into anti-gay attitudes: "Homosexuals have the right to run for office", and "Same-sex couples have the right to marry". Respondents indicated their level of approval with these statements on 10-point Likert scales ranging from 1 ("Strongly disapprove") to 10 ("Strongly approve"). Both items were highly correlated ( $r=$ $.57, p<.001)$, and after reverse-coding, they yielded a scale with $M=6.61(S D=3.30)$.

\section{Data analytic procedure}

In all studies, we performed multilevel modeling with respondents (individual level) nested within regions (context level). All predictors were grand-mean centered. We first estimated empty (intercept-only) models which provide insight in the variances at the individual and contextual level. We assessed intraclass correlations (ICC) to ensure there was substantial between-level variance. The ICC for anti-gay prejudice was 0.24 . Next, all predictors were added to the model. At the individual level, we tested the association of religion with anti-gay prejudice. At the contextual level, we tested the effect of each cultural dimension separately. Specifically, we included the relationship of power distance, uncertainty avoidance, masculinity, or individualism with anti-gay prejudice, while controlling for individual differences in religious affiliation. Finally, we analyzed the slope variance, testing whether the relationship between religion and anti-gay prejudice significantly differed across countries, and whether any cultural dimension explained (part of) the variance in the slopes. We used full-information maximum-likelihood estimates with robust standard errors, modelling a random coefficient model (Raudenbush \& Bryk, 2002) where intercept and slope coefficients vary across countries. In all studies, the large sample sizes ensure sufficient statistical power to detect even small main effects, although the number 
of contextual units in Study 1 (i.e., 20) reduces the power of finding cross-level interaction effects (Hox, 2013).

\section{Results}

Table 1 summarizes the results of all cross-level interaction analyses. At the individual level, religion was significantly and positively related to anti-gay sentiment. At the contextual level, uncertainty avoidance was positively related to anti-gay sentiment, indicating that antigay attitudes tended to be higher in countries high in uncertainty avoidance. Most importantly, three marginally significant interaction effects emerged. Multilevel simple slope analyses showed that the relationship between religion and anti-gay prejudice was weaker in countries high in power distance, compared to countries low in power distance (see Table 2). Even further, the association of religion with anti-gay prejudice was not significant in countries very high (i.e., $2 \mathrm{SD}$ above the mean) in power distance, it was slightly stronger in countries high (i.e., $1 \mathrm{SD}$ above the mean) in power distance, somewhat stronger in low (i.e., $1 \mathrm{SD}$ below the mean) power distance countries, and it was strongest in countries very low (i.e., 2 SD below the mean) in power distance.

Similarly, the relationship between religion and anti-gay prejudice was stronger in countries low in uncertainty avoidance, as opposed to countries high in uncertainty avoidance, and it was not significant in countries very high in uncertainty avoidance. Thirdly, the religion-anti-gay prejudice relationship was strongest in countries (very) high in masculinity, and was weakest and even absent in countries (very) low in masculinity. Finally, the religionanti-gay prejudice association was stronger in countries high in individualism, as opposed to countries low in individualism. In very collectivistic countries (i.e., very low in individualism), the association was absent. 
In sum, the association between being religious and anti-gay prejudice seems less pronounced and even absent in countries high on power distance, high on masculinity, and low on individualism. There is a similar trend of weaker relationships in high uncertainty avoidance countries, but the cross-level interaction term was not statistically significant.

\section{Study 2}

Study 2 examined religiosity as a continuous, individual difference predictor of prejudice. Further, we investigated an additional form of prejudice (i.e., sexist attitudes towards women) and we did cross-country comparisons worldwide (instead of within the Americas).

\section{Method}

\section{Participants}

We used data from the sixth round of the World Values Survey (WVS, 2014, http://www.worldvaluessurvey.org). WVS is a global scientific network studying socialpolitical life. WVS has made substantial efforts to ensure the equivalence of comparative data. Round 6 data were collected in representative national samples between 2010 and 2014. For 15 countries (Algeria, Azerbaijan, Armenia, Belarus, Cyprus, Georgia, Palestine, Haiti, Kazakhstan, Kyrgyzstan, Rwanda, Tunisia, Zimbabwe, Uzbekistan, and Yemen), cultural scores were unavailable, these were omitted (see Table 1 in SOM for all included countries and their cultural scores). The final sample comprised 69,798 individuals from 45 countries $\left(M_{\mathrm{age}}=42.43, S D_{\text {age }}=16.64\right.$, with $50.7 \%$ women; $M=1,551$ observations per country $)$.

\section{Measures}

Religious affiliation. Twenty-one percent of the sample had no religious affiliation, 19.4\% identified as (Roman) Catholic, $12.8 \%$ as Muslim, $6.7 \%$ as Orthodox, 5.9\% Protestant, 
5.6\% Hindu, 5.5\% Buddhist, 4.5\% Sunni, 2.0\% Evangelical, and 16.6\% adhered to another religion. We dummy-coded this variable with 0 ("No religion") and 1 (“A religion").

Religiosity. We created a religiosity scale using the items "How often do you attend religious services apart from special occasions", and "How often do you pray apart from at religious services". The first item was answered on a 7-point Likert scale ranging from 1 ("More than once a week") to 7 ("Never"), the second on an 8-point scale anchored by 1 ("Several times a day") to 8 ("Never"). Both items were positively interrelated ( $r=.67, p<$ $.001)$. After reverse-coding and standardizing them, a scale was formed $(M=-0.01 ; S D=$ $0.92)$.

Cultural dimensions. Scores were retrieved from https://www.hofstede-insights.com (see Table B in SOM).

Anti-gay prejudice. Participants responded to the statement "Homosexuality is justifiable" using a 10-point scale ranging from 1 ("Never justifiable") to 10 ("Always justifiable"). The item was reverse-coded, with $M=7.37(S D=3.18)$.

Sexist attitudes. Similar to Van Assche and colleagues (2017), six sexism items were used. The first two read "When jobs are scarce, men should have more right to a job than women", and "If a woman earns more money than her husband, it's almost certain to cause problems". Respondents selected 1 ("Agree"), 2 ("Neither"), or 3 ("Disagree"). The other four items ("When a mother works for pay, the children suffer", "On the whole, men make better political leaders than women do", "A university education is more important for a boy than for a girl", and "On the whole, men make better business executives than women do") were answered on 4-point scales ranging from 1 ("Strongly agree") to 4 ("Strongly disagree"). All items were reverse-coded and, after standardizing, they yielded a reliable scale with $\alpha=.77 ; M=-0.05(S D=0.67)$. Both individual-level outcomes (i.e., anti-gay prejudice 
and sexism) were positively interrelated $(r=.36, p<.001)$, and both positively correlated with religiosity $(r=.30, p<.001$; and $r=.22, p<.001$, for anti-gay prejudice and sexism, respectively).

\section{Results}

We used the same procedure as in Study 1. The ICC was 0.35 for anti-gay prejudice and 0.29 for sexism. Tables 1 and 2 summarize all main results. First, religion and religiosity were significantly and positively related to anti-gay sentiment and sexist attitudes. Second, power distance was positively related to anti-gay prejudice and sexism, whereas individualism showed an opposite relation. There was also a trend of higher sexism levels in countries high in masculinity. Most importantly, some significant cross-level interaction effects emerged.

Simple slopes indicated that the associations of religion and religiosity with anti-gay prejudice and sexism were not significant in countries very high (i.e., 2 SD above the mean) in power distance, slightly stronger in countries high (i.e., $1 \mathrm{SD}$ above the mean) in power distance, somewhat stronger in low (i.e., 1 SD below the mean) power distance countries, and strongest in countries very low (i.e., $2 \mathrm{SD}$ below the mean) in power distance.

Similar trends were found for uncertainty avoidance and masculinity. Moreover, as in Study 1 , the relationships between religion or religiosity and prejudice were stronger in countries high in individualism, as opposed to countries low in individualism. In very collectivistic countries, the religion-anti-gay prejudice relation was absent. In sum, the association between being religious and being prejudiced was less pronounced and even absent in countries high in power distance and collectivism. There was a similar trend of weaker relationships in countries high on uncertainty avoidance and masculinity, but these interaction terms were not statistically significant. 
Table 1. Unstandardized estimates [95\% confidence intervals in brackets] of multilevel regression analyses on anti-gay prejudice and sexism in the Americas Barometer (AB; Study 1), the World Value Survey (WVS; Study 2), and the European Social Survey (ESS; Study 3).

\begin{tabular}{|c|c|c|c|c|}
\hline AB: Religion - Homophobia & Power Distance & Uncertainty Avoidance & Masculinity & Individualism \\
\hline \multirow{2}{*}{ Religion ( 1 = yes $)$} & $0.518 ; p<.001$ & $0.508 ; p<.001$ & $0.515 ; p<.001$ & $0.543 ; p<.001$ \\
\hline & {$[0.354 ; 0.681]$} & {$[0.332 ; 0.685]$} & {$[0.347 ; 0.684]$} & {$[0.369 ; 0.717]$} \\
\hline \multirow{2}{*}{ Contextual Moderator } & $0.020 ; p=.388$ & $0.039 ; p=.019$ & $0.018 ; p=.488$ & $-0.045 ; p=.128$ \\
\hline & {$[-0.028 ; 0.068]$} & {$[0.007 ; 0.071]$} & {$[-0.035 ; 0.070]$} & {$[-0.104 ; 0.014]$} \\
\hline \multirow{2}{*}{ Religion $\times$ Context } & $-0.010 ; p=.055$ & $-0.006 ; p=.127$ & $-0.011 ; p=.053$ & $0.014 ; p=.061$ \\
\hline & {$[-0.020 ; 0.000]$} & {$[-0.014 ; 0.002]$} & {$[-0.022 ; 0.000]$} & {$[-0.001 ; 0.028]$} \\
\hline WVS: Religion - Homophobia & Power Distance & Uncertainty Avoidance & Masculinity & Individualism \\
\hline \multirow{2}{*}{ Religion ( $1=$ yes $)$} & $1.003 ; p<.001$ & $1.061 ; p<.001$ & $1.062 ; p<.001$ & $1.057 ; p<.001$ \\
\hline & {$[0.733 ; 1.273]$} & {$[0.777 ; 1.346]$} & {$[0.771 ; 1.352]$} & {$[0.798 ; 1.312]$} \\
\hline \multirow{2}{*}{ Contextual Moderator } & $0.067 ; p<.001$ & $0.005 ; p=.733$ & $0.028 ; p=.111$ & $-0.057 ; p<.001$ \\
\hline & {$[0.042 ; 0.923]$} & {$[-0.023 ; 0.033]$} & {$[-0.007 ; 0.063]$} & {$[-0.079 ;-0.035]$} \\
\hline \multirow{2}{*}{ Religion $\times$ Context } & $-0.013 ; p=.071$ & $-0.007 ; p=.230$ & $-0.004 ; p=.576$ & $0.013 ; p=.025$ \\
\hline & {$[-0.026 ; 0.001]$} & {$[-0.020 ; 0.005]$} & {$[-0.021 ; 0.012]$} & {$[0.002 ; 0.024]$} \\
\hline WVS: Religion - Sexism & Power Distance & Uncertainty Avoidance & Masculinity & Individualism \\
\hline \multirow{2}{*}{ Religion ( $1=$ yes $)$} & $0.078 ; p<.001$ & $0.088 ; p<.001$ & $0.088 ; p<.001$ & $0.086 ; p<.001$ \\
\hline & {$[0.042 ; 0.113]$} & {$[0.051 ; 0.125]$} & {$[0.051 ; 0.125]$} & {$[0.051 ; 0.122]$} \\
\hline \multirow{2}{*}{ Contextual Moderator } & $0.012 ; p<.001$ & $0.001 ; p=.785$ & $0.006 ; p=.064$ & $-0.007 ; p=.001$ \\
\hline & {$[0.008 ; 0.016]$} & {$[-0.004 ; 0.005]$} & {$[0.000 ; 0.012]$} & {$[-0.012 ;-0.003]$} \\
\hline \multirow{2}{*}{ Religion $\times$ Context } & $-0.002 ; p=.041$ & $0.000 ; p=.593$ & $0.000 ; p=.999$ & $0.001 ; p=.188$ \\
\hline & {$[-0.004 ;-0.001]$} & {$[-0.002 ; 0.001]$} & {$[-0.002 ; 0.002]$} & {$[-0.001 ; 0.002]$} \\
\hline WVS: Religiosity - Homophobia & Power Distance & Uncertainty Avoidance & Masculinity & Individualism \\
\hline \multirow{2}{*}{ Religiosity } & $0.488 ; p<.001$ & $0.503 ; p<.001$ & $0.501 ; p<.001$ & $0.519 ; p<.001$ \\
\hline & {$[0.372 ; 0.604]$} & {$[0.368 ; 0.639]$} & {$[0.366 ; 0.636]$} & {$[0.403 ; 0.635]$} \\
\hline \multirow{2}{*}{ Contextual Moderator } & $0.052 ; p<.001$ & $0.002 ; p=.887$ & $0.018 ; p=.226$ & $-0.043 ; p<.001$ \\
\hline & {$[0.030 ; 0.074]$} & {$[-0.022 ; 0.025]$} & {$[-0.012 ; 0.049]$} & {$[-0.062 ;-0.023]$} \\
\hline \multirow{2}{*}{ Religion $\times$ Context } & $-0.012 ; p<.001$ & $-0.003 ; p=.276$ & $-0.005 ; p=.263$ & $0.011 ; p<.001$ \\
\hline & {$[-0.019 ;-0.006]$} & {$[-0.010 ; 0.003]$} & {$[-0.013 ; 0.004]$} & {$[0.005 ; 0.016]$} \\
\hline WVS: Religiosity - Sexism & Power Distance & Uncertainty Avoidance & Masculinity & Individualism \\
\hline \multirow{2}{*}{ Religiosity } & $0.037 ; p=.002$ & $0.038 ; p<.001$ & $0.038 ; p=.002$ & $0.040 ; p=.001$ \\
\hline & {$[0.014 ; 0.061]$} & {$[0.015 ; 0.062]$} & {$[0.015 ; 0.062]$} & {$[0.017 ; 0.062]$} \\
\hline \multirow{2}{*}{ Contextual Moderator } & $0.010 ; p<.001$ & $0.001 ; p=.799$ & $0.005 ; p=.096$ & $-0.007 ; p=.003$ \\
\hline & {$[0.005 ; 0.014]$} & {$[-0.004 ; 0.005]$} & {$[0.000 ; 0.011]$} & {$[-0.011 ;-0.002]$} \\
\hline \multirow{2}{*}{ Religion $\times$ Context } & $0.000 ; p=.430$ & $0.000 ; p=.804$ & $0.000 ; p=.996$ & $0.001 ; p=.045$ \\
\hline & {$[-0.002 ; 0.000]$} & {$[-0.001 ; 0.001]$} & {$[-0.001 ; 0.001]$} & {$[0.001 ; 0.002]$} \\
\hline
\end{tabular}




\begin{tabular}{|c|c|c|c|c|}
\hline ESS: Religion - Homophobia & Power Distance & Uncertainty Avoidance & Masculinity & Individualism \\
\hline \multirow{2}{*}{ Religion $(1=$ yes $)$} & $0.373 ; p<.001$ & $0.378 ; p<.001$ & $0.375 ; p<.001$ & $0.377 ; p<.001$ \\
\hline & {$[0.342 ; 0.404]$} & {$[0.348 ; 0.408]$} & {$[0.344 ; 0.405]$} & {$[0.347 ; 0.408]$} \\
\hline \multirow{2}{*}{ Contextual Moderator } & $0.684 ; p<.001$ & $0.851 ; p<.001$ & $1.045 ; p<.001$ & $-0.696 ; p<.001$ \\
\hline & {$[0.541 ; 0.826]$} & {$[0.708 ; 0.994]$} & {$[0.916 ; 1.174]$} & {$[-0.919 ;-0.472]$} \\
\hline \multirow{2}{*}{ Religion $\times$ Context } & $-0.023 ; p=.473$ & $-0.019 ; p=.586$ & $-0.061 ; p=.080$ & $0.133 ; p=.005$ \\
\hline & {$[-0.088 ; 0.041]$} & {$[-0.089 ; 0.050]$} & {$[-0.007 ; 0.129]$} & {$[0.039 ; 0.226]$} \\
\hline ESS: Religion - Sexism & Power Distance & Uncertainty Avoidance & Masculinity & Individualism \\
\hline \multirow{2}{*}{ Religion ( 1 = yes $)$} & $0.190 ; p<.001$ & $0.179 ; p<.001$ & $0.173 ; p<.001$ & $0.186 ; p<.001$ \\
\hline & {$[0.159 ; 0.221]$} & {$[0.150 ; 0.209]$} & {$[0.143 ; 0.202]$} & {$[0.157 ; 0.215]$} \\
\hline \multirow{2}{*}{ Contextual Moderator } & $0.424 ; p<.001$ & $0.643 ; p<.001$ & $0.819 ; p<.001$ & $-0.319 ; p<.001$ \\
\hline & {$[0.319 ; 0.529]$} & {$[0.541 ; 0.745]$} & {$[0.732 ; 0.906]$} & {$[-0.485 ;-0.153]$} \\
\hline \multirow{2}{*}{ Religion $\times$ Context } & $-0.067 ; p=.039$ & $-0.091 ; p=.009$ & $-0.148 ; p<.001$ & $0.151 ; p=.001$ \\
\hline & {$[-0.130 ;-0.003]$} & {$[-0.160 ;-0.023]$} & {$[-0.215 ;-0.081]$} & {$[0.061 ; 0.242]$} \\
\hline ESS: Religiosity - Homophobia & Power Distance & Uncertainty Avoidance & Masculinity & Individualism \\
\hline \multirow{2}{*}{ Religiosity } & $0.272 ; p<.001$ & $0.272 ; p<.001$ & $0.267 ; p<.001$ & $0.273 ; p<.001$ \\
\hline & {$[0.250 ; 0.292]$} & {$[0.252 ; 0.292]$} & {$[0.247 ; 0.287]$} & {$[0.253 ; 0.293]$} \\
\hline \multirow{2}{*}{ Contextual Moderator } & $0.676 ; p<.001$ & $0.830 ; p<.001$ & $0.971 ; p<.001$ & $-0.569 ; p<.001$ \\
\hline & {$[0.543 ; 0.809]$} & {$[0.696 ; 0.964]$} & {$[0.848 ; 1.093]$} & {$[-0.781 ;-0.356]$} \\
\hline \multirow{2}{*}{ Religiosity $\times$ Context } & $-0.044 ; p=.047$ & $-0.057 ; p=.017$ & $-0.077 ; p=.001$ & $0.064 ; p=.037$ \\
\hline & {$[-0.087 ;-0.001]$} & {$[-0.103 ;-0.010]$} & {$[-0.122 ;-0.031]$} & {$[0.004 ; 0.124]$} \\
\hline ESS: Religiosity - Sexism & Power Distance & Uncertainty Avoidance & Masculinity & Individualism \\
\hline \multirow{2}{*}{ Religiosity } & $0.151 ; p<.001$ & $0.146 ; p<.001$ & $0.142 ; p<.001$ & $0.150 ; p<.001$ \\
\hline & {$[0.131 ; 0.170]$} & {$[0.127 ; 0.166]$} & {$[0.123 ; 0.162]$} & {$[0.131 ; 0.169]$} \\
\hline \multirow{2}{*}{ Contextual Moderator } & $0.370 ; p<.001$ & $0.570 ; p<.001$ & $0.707 ; p<.001$ & $-0.205 ; p=.009$ \\
\hline & {$[0.272 ; 0.468]$} & {$[0.475 ; 0.665]$} & {$[0.626 ; 0.789]$} & {$[-0.359 ;-0.050]$} \\
\hline \multirow{2}{*}{ Religiosity $\times$ Context } & $-0.037 ; p=.080$ & $-0.046 ; p=.049$ & $-0.062 ; p=.007$ & $0.124 ; p<.001$ \\
\hline & {$[-0.078 ; 0.004]$} & {$[-0.092 ;-0.001]$} & {$[-0.108 ;-0.017]$} & {$[0.068 ; 0.181]$} \\
\hline
\end{tabular}


Table 2. Simple slope effects [ $95 \%$ confidence intervals in brackets] of being religious on anti-gay prejudice and sexism at different levels of Hofstede's cultural dimensions in the Americas Barometer (AB), World Value Survey (WVS), and European Social Survey (ESS).

\begin{tabular}{|c|c|c|c|c|}
\hline AB: Religion - Homophobia & Power Distance & Uncertainty Avoidance & Masculinity & Individualism \\
\hline \multirow{2}{*}{ Very low (- $2 \mathrm{SD})$} & $0.860 ; p<.001$ & $0.783 ; p=.001$ & $0.838 ; p<.001$ & $0.063 ; p=.796$ \\
\hline & {$[0.474 ; 1.245]$} & {$[0.391 ; 1.176]$} & {$[0.470 ; 1.207]$} & {$[-0.444 ; 0.571]$} \\
\hline \multirow{2}{*}{ Low (- $1 \mathrm{SD})$} & $0.689 ; p<.001$ & $0.646 ; p<.001$ & $0.677 ; p<.001$ & $0.303 ; p=.038$ \\
\hline & {$[0.450 ; 0.928]$} & {$[0.402 ; 0.890]$} & {$[0.442 ; 0.912]$} & {$[0.019 ; 0.587]$} \\
\hline \multirow{2}{*}{ High (+ 1 SD) } & $0.347 ; p=.008$ & $0.371 ; p=.009$ & $0.354 ; p=.006$ & $0.783 ; p<.001$ \\
\hline & {$[0.108 ; 0.589]$} & {$[0.109 ; 0.633]$} & {$[0.119 ; 0.589]$} & {$[0.456 ; 1.109]$} \\
\hline \multirow{2}{*}{ Very high (+ 2 SD) } & $0.176 ; p=.345$ & $0.233 ; p=.248$ & $0.193 ; p=.285$ & $1.022 ; p=.001$ \\
\hline & {$[-0.210 ; 0.562]$} & {$[-0.182 ; 0.649]$} & {$[-0.177 ; 0.562]$} & {$[0.466 ; 1.579]$} \\
\hline WVS: Religion - Homophobia & Power Distance & Uncertainty Avoidance & Masculinity & Individualism \\
\hline \multirow{2}{*}{ Very low (- 2 SD) } & $1.476 ; p<.001$ & $1.386 ; p<.001$ & $1.211 ; p<.001$ & $0.498 ; p=.073$ \\
\hline & {$[0.938 ; 2.013]$} & {$[0.784 ; 1.987]$} & {$[0.627 ; 1.795]$} & {$[-0.049 ; 1.045]$} \\
\hline \multirow{2}{*}{ Low (- $1 \mathrm{SD})$} & $1.239 ; p<.001$ & $1.223 ; p<.001$ & $1.136 ; p<.001$ & $0.778 ; p<.001$ \\
\hline & {$[0.901 ; 1.578]$} & {$[0.839 ; 1.608]$} & {$[0.763 ; 1.510]$} & {$[0.423 ; 1.132]$} \\
\hline \multirow{2}{*}{$\operatorname{High}(+1 \mathrm{SD})$} & $0.766 ; p=.001$ & $0.899 ; p<.001$ & $0.989 ; p<.001$ & $1.337 ; p<.001$ \\
\hline & {$[0.361 ; 1.171]$} & {$[0.497 ; 1.301]$} & {$[0.568 ; 1.406]$} & {$[0.983 ; 1.691]$} \\
\hline \multirow{2}{*}{ Very high $(+2$ SD) } & $0.530 ; p=.093$ & $0.737 ; p=.022$ & $0.912 ; p=.007$ & $1.617 ; p<.001$ \\
\hline & {$[-0.093 ; 1.153]$} & {$[0.113 ; 1.360]$} & {$[0.270 ; 1.555]$} & {$[1.070 ; 2.164]$} \\
\hline WVS: Religion - Sexism & Power Distance & Uncertainty Avoidance & Masculinity & Individualism \\
\hline \multirow{2}{*}{ Very low (- 2 SD) } & $0.148 ; p<.001$ & $0.106 ; p=.001$ & $0.088 ; p=.002$ & $0.044 ; p=.237$ \\
\hline & {$[0.079 ; 0.217]$} & {$[0.027 ; 0.186]$} & {$[0.015 ; 0.161]$} & {$[-0.031 ; 0.119]$} \\
\hline \multirow{2}{*}{ Low (- $1 \mathrm{SD})$} & $0.113 ; p<.001$ & $0.097 ; p=.001$ & $0.088 ; p=.001$ & $0.065 ; p=.011$ \\
\hline & {$[0.070 ; 0.156]$} & {$[0.046 ; 0.148]$} & {$[0.041 ; 0.135]$} & {$[0.016 ; 0.114]$} \\
\hline \multirow{2}{*}{$\operatorname{High}(+1 \mathrm{SD})$} & $0.043 ; p=.119$ & $0.079 ; p=.003$ & $0.088 ; p=.002$ & $0.108 ; p<.001$ \\
\hline & {$[-0.012 ; 0.097]$} & {$[0.030 ; 0.128]$} & {$[0.036 ; 0.141]$} & {$[0.060 ; 0.155]$} \\
\hline \multirow{2}{*}{ Very high $(+2 \mathrm{SD})$} & $0.007 ; p=.857$ & $0.070 ; p=.073$ & $0.088 ; p=.032$ & $0.129 ; p=.001$ \\
\hline & {$[-0.076 ; 0.090]$} & {$[-0.007 ; 0.147]$} & {$[0.008 ; 0.169]$} & {$[0.056 ; 0.202]$} \\
\hline WVS: Religiosity - Homophobia & Power Distance & Uncertainty Avoidance & Masculinity & Individualism \\
\hline \multirow{2}{*}{ Very low (- 2 SD) } & $0.952 ; p<.001$ & $0.652 ; p<.001$ & $0.652 ; p<.001$ & $0.059 ; p=.636$ \\
\hline & {$[0.704 ; 1.200]$} & {$[0.355 ; 0.949]$} & {$[0.362 ; 0.942]$} & {$[-0.192 ; 0.311]$} \\
\hline \multirow{2}{*}{ Low (- $1 \mathrm{SD})$} & $0.720 ; p<.001$ & $0.578 ; p<.001$ & $0.576 ; p<.001$ & $0.301 ; p<.001$ \\
\hline & {$[0.564 ; 0.876]$} & {$[0.391 ; 0.764]$} & {$[0.394 ; 0.759]$} & {$[0.146 ; 0.457]$} \\
\hline \multirow{2}{*}{$\operatorname{High}(+1 \mathrm{SD})$} & $0.256 ; p=.004$ & $0.429 ; p<.001$ & $0.425 ; p<.001$ & $0.762 ; p<.001$ \\
\hline & {$[0.086 ; 0.426]$} & {$[0.232 ; 0.626]$} & {$[0.227 ; 0.624]$} & {$[0.597 ; 0.927]$} \\
\hline \multirow{2}{*}{ Very high (+ 2 SD) } & $0.024 ; p=.856$ & $0.355 ; p=.026$ & $0.350 ; p=.028$ & $0.979 ; p<.001$ \\
\hline & {$[-0.242 ; 0.290]$} & {$[0.044 ; 0.665]$} & {$[0.039 ; 0.661]$} & {$[0.718 ; 1.241]$} \\
\hline
\end{tabular}




\begin{tabular}{|c|c|c|c|c|}
\hline WVS: Religiosity - Sexism & Power Distance & Uncertainty Avoidance & Masculinity & Individualism \\
\hline \multirow{2}{*}{ Very low (- $2 \mathrm{SD})$} & $0.056 ; p=.031$ & $0.044 ; p=.092$ & $0.038 ; p=.137$ & $-0.006 ; p=.803$ \\
\hline & {$[0.005 ; 0.106]$} & {$[-0.008 ; 0.095]$} & {$[-0.013 ; 0.089]$} & {$[-0.055 ; 0.043]$} \\
\hline \multirow{2}{*}{ Low (- $1 \mathrm{SD})$} & $0.047 ; p=.005$ & $0.041 ; p=.014$ & $0.038 ; p=.020$ & $0.017 ; p=.276$ \\
\hline & {$[0.015 ; 0.078]$} & {$[0.009 ; 0.073]$} & {$[0.006 ; 0.070]$} & {$[-0.014 ; 0.047]$} \\
\hline \multirow{2}{*}{ High (+ $1 \mathrm{SD})$} & $0.028 ; p=.105$ & $0.035 ; p=.047$ & $0.038 ; p=.032$ & $0.062 ; p<.001$ \\
\hline & {$[-0.006 ; 0.063]$} & {$[0.001 ; 0.070]$} & {$[0.003 ; 0.073]$} & {$[0.030 ; 0.095]$} \\
\hline \multirow{2}{*}{ Very high (+ 2 SD) } & $0.019 ; p=.482$ & $0.032 ; p=.241$ & $0.038 ; p=.165$ & $0.085 ; p=.002$ \\
\hline & {$[-0.035 ; 0.073]$} & {$[-0.022 ; 0.087]$} & {$[-0.016 ; 0.093]$} & {$[0.034 ; 0.136]$} \\
\hline ESS: Religion - Homophobia & Power Distance & Uncertainty Avoidance & Masculinity & Individualism \\
\hline \multirow{2}{*}{ Very low (- $2 \mathrm{SD})$} & $0.394 ; p<.001$ & $0.393 ; p<.001$ & $0.428 ; p<.001$ & $0.291 ; p<.001$ \\
\hline & {$[0.329 ; 0.460]$} & {$[0.327 ; 0.459]$} & {$[0.366 ; 0.489]$} & {$[0.221 ; 0.360]$} \\
\hline \multirow{2}{*}{ Low (- $1 \mathrm{SD})$} & $0.384 ; p<.001$ & $0.385 ; p<.001$ & $0.401 ; p<.001$ & $0.334 ; p<.001$ \\
\hline & {$[0.341 ; 0.426]$} & {$[0.343 ; 0.428]$} & {$[0.363 ; 0.439]$} & {$[0.290 ; 0.378]$} \\
\hline \multirow{2}{*}{ High (+ 1 SD) } & $0.363 ; p<.001$ & $0.370 ; p<.001$ & $0.348 ; p<.001$ & $0.421 ; p<.001$ \\
\hline & {$[0.320 ; 0.405]$} & {$[0.330 ; 0.409]$} & {$[0.301 ; 0.394]$} & {$[0.379 ; 0.462]$} \\
\hline \multirow{2}{*}{ Very high $(+2 \mathrm{SD})$} & $0.352 ; p<.001$ & $0.362 ; p<.001$ & $0.321 ; p<.001$ & $0.464 ; p<.001$ \\
\hline & {$[0.286 ; 0.418]$} & {$[0.300 ; 0.424]$} & {$[0.249 ; 0.393]$} & {$[0.398 ; 0.531]$} \\
\hline ESS: Religion - Sexism & Power Distance & Uncertainty Avoidance & Masculinity & Individualism \\
\hline \multirow{2}{*}{ Very low (- $2 \mathrm{SD})$} & $0.250 ; p<.001$ & $0.254 ; p<.001$ & $0.303 ; p<.001$ & $0.087 ; p=.012$ \\
\hline & {$[0.186 ; 0.315]$} & {$[0.193 ; 0.315]$} & {$[0.242 ; 0.364]$} & {$[0.020 ; 0.155]$} \\
\hline \multirow{2}{*}{ Low (- $1 \mathrm{SD})$} & $0.220 ; p<.001$ & $0.217 ; p<.001$ & $0.238 ; p<.001$ & $0.137 ; p<.001$ \\
\hline & {$[0.179 ; 0.262]$} & {$[0.178 ; 0.255]$} & {$[0.200 ; 0.275]$} & {$[0.094 ; 0.180]$} \\
\hline \multirow{2}{*}{ High (+ $1 \mathrm{SD})$} & $0.160 ; p<.001$ & $0.142 ; p<.001$ & $0.108 ; p<.001$ & $0.236 ; p<.001$ \\
\hline & {$[0.117 ; 0.202]$} & {$[0.100 ; 0.184]$} & {$[0.062 ; 0.153]$} & {$[0.196 ; 0.276]$} \\
\hline \multirow{2}{*}{ Very high $(+2 \mathrm{SD})$} & $0.130 ; p<.001$ & $0.105 ; p=.002$ & $0.043 ; p=.235$ & $0.285 ; p<.001$ \\
\hline & {$[0.064 ; 0.195]$} & {$[0.040 ; 0.170]$} & {$[-0.028 ; 0.114]$} & {$[0.221 ; 0.350]$} \\
\hline ESS: Religiosity - Homophobia & Power Distance & Uncertainty Avoidance & Masculinity & Individualism \\
\hline \multirow{2}{*}{ Very low (- $2 \mathrm{SD})$} & $0.311 ; p<.001$ & $0.318 ; p<.001$ & $0.334 ; p<.001$ & $0.231 ; p<.001$ \\
\hline & {$[0.265 ; 0.357]$} & {$[0.275 ; 0.361]$} & {$[0.292 ; 0.377]$} & {$[0.187 ; 0.275]$} \\
\hline \multirow{2}{*}{ Low (- $1 \mathrm{SD})$} & $0.291 ; p<.001$ & $0.294 ; p<.001$ & $0.301 ; p<.001$ & $0.252 ; p<.001$ \\
\hline & {$[0.262 ; 0.321]$} & {$[0.267 ; 0.322]$} & {$[0.275 ; 0.327]$} & {$[0.224 ; 0.280]$} \\
\hline \multirow{2}{*}{ High (+ $1 \mathrm{SD})$} & $0.252 ; p<.001$ & $0.249 ; p<.001$ & $0.233 ; p<.001$ & $0.294 ; p<.001$ \\
\hline & {$[0.224 ; 0.279]$} & {$[0.221 ; 0.276]$} & {$[0.203 ; 0.264]$} & {$[0.266 ; 0.322]$} \\
\hline \multirow{2}{*}{ Very high $(+2 \mathrm{SD})$} & $0.232 ; p<.001$ & $0.226 ; p<.001$ & $0.200 ; p<.001$ & $0.315 ; p<.001$ \\
\hline & {$[0.189 ; 0.275]$} & {$[0.183 ; 0.268]$} & {$[0.152 ; 0.247]$} & {$[0.271 ; 0.359]$} \\
\hline ESS: Religiosity - Sexism & Power Distance & Uncertainty Avoidance & Masculinity & Individualism \\
\hline \multirow{2}{*}{ Very low (- $2 \mathrm{SD})$} & $0.184 ; p<.001$ & $0.184 ; p<.001$ & $0.197 ; p<.001$ & $0.069 ; p=.001$ \\
\hline & {$[0.141 ; 0.227]$} & {$[0.142 ; 0.226]$} & {$[0.155 ; 0.239]$} & {$[0.027 ; 0.110]$} \\
\hline Low (- $1 \mathrm{SD})$ & $0.167 ; p<.001$ & $0.165 ; p<.001$ & $0.170 ; p<.001$ & $0.109 ; p<.001$ \\
\hline
\end{tabular}




\begin{tabular}{lcccc} 
& {$[0.139 ; 0.195]$} & {$[0.138 ; 0.192]$} & {$[0.144 ; 0.196]$} & {$[0.083 ; 0.136]$} \\
High (+ 1 SD) & $0.134 ; p<.001$ & $0.128 ; p<.001$ & $0.115 ; p<.001$ & $0.191 ; p<.001$ \\
& {$[0.108 ; 0.160]$} & {$[0.101 ; 0.155]$} & {$[0.085 ; 0.145]$} & {$[0.164 ; 0.217]$} \\
Very high (+ 2 SD) & $0.118 ; p<.001$ & $0.109 ; p<.001$ & $0.088 ; p<.001$ & $0.231 ; p<.001$ \\
& {$[0.076 ; 0.159]$} & {$[0.067 ; 0.151]$} & {$[0.041 ; 0.134]$} & {$[0.190 ; 0.273]$} \\
\hline
\end{tabular}

Note: In the AB and the WVS, there were no countries very high (+ $2 \mathrm{SD})$ in

uncertainty avoidance; and there were no countries at the very low (- 2 SD) end of the individualism continuum in the $\mathrm{AB}$. As such, these slopes should be interpreted with caution, as they fall outside the range of countries included in those specific datasets.

\section{Study 3}

Study 3 aimed to replicate Study 1 and 2 at a more fine-grained contextual level (i.e., regions within Europe).

\section{Method}

\section{Participants}

Study 3 analyzed data from the eighth round of the European Social Survey (ESS, 2016, http://www.europeansocialsurvey.org). Data were collected by face-to-face interviews and are representative for each country. Round 8 data were collected between September and December 2016 in 23 European countries. Within each country, NUTS ("Nomenclature des Unités Territoriales Statistiques") can be distinguished. These units delineate within-country regions according to socioeconomic, cultural and historical characteristics (ESS, 2016). NUTS-regions were used as higher-level units for this study. The sample consisted of 44,386 individuals from 274 NUTS-regions $\left(M_{\text {age }}=49.14, S D_{\text {age }}=18.61\right.$, with 52.6\% women; $M=$ 162 observations per country).

\section{Measures}

Religious affiliation. Forty percent of the sample had no religious affiliation, $33.9 \%$ identified as (Roman) Catholic, $11.5 \%$ as Protestant, $4.5 \%$ as Jewish, $4.3 \%$ as (Eastern) 
Orthodox, 3.1\% Muslim, and 2.7\% adhered to another religion We dummy-coded this variable with 0 (“No religion") and 1 (“A religion").

Religiosity. We created a religiosity scale using the following items: "How religious are you", "How often do you attend religious services apart from special occasions", and "How often do you pray apart from at religious services". The first item was answered on an 11-point Likert scale ranging from 0 ("Not at all religious") to 10 ("Very religious"). The other two items were answered on 7-point scales anchored by 1 ("Every day") to 7 ("Never"). The latter two items were reverse-coded, and after standardizing all three items, our scale was reliable $(\alpha=.85 ; M=0.00 ; S D=0.88)$.

Cultural dimensions. Kaasa, Vadi, and Varblane (2014) showed that ESS can be useful for measuring culture, and they extract indicators for Hofstede's dimensions in European NUTS-regions (see Table B in SOM for means, standard deviations, and correlations between the dimensions). For power distance, four items were used (and reversecoded). The first three items were rated on 11-point scales: "How satisfied are you with the way democracy works in your country?" (0 = "Extremely dissatisfied"; 10 = "Extremely satisfied"); "To what extent are you allowed to decide how your daily work is organized?" (0 = "I have/had no influence"; 10 = "I have/had complete control"), and "To what extent do you trust your country's parliament?" ( $0=$ "No trust at all"; $10=$ "Complete trust"). The fourth item read "The government should reduce differences in income levels", and was answered on a 5-point scale anchored by 1 (“Agree strongly") and 5 (“Disagree strongly").

For uncertainty avoidance, five items were administered (and reverse-coded). The first three items were rated on 6-point scales anchored by 1 ("Very much like me") and 6 ("Not like me at all"): "It is important to live in secure and safe surroundings", "It is important that our government is strong and ensures safety", and "It is important to follow traditions and 
customs". The last two items were rated on 11-point scales: "Most people can be trusted or you can't be too careful" ( 0 = "You can't be too careful"; $10=$ "Most people can be trusted"), and "Immigrants make our country a worse or better place to live" $(0=$ "Worse place to live"; $10=$ "Better place to live").

Four items tapped into masculinity. The first three items were rated on 6-point scales ranging from 1 ("Very much like me") to 6 ("Not like me at all"): "It is important to be rich, have money and expensive things", "It is important to show your abilities and be admired", and "It is important to be successful and that people recognize achievements". The last item, "Men should have more right to job than women when jobs are scarce", was rated on a scale ranging from 1 (“Agree strongly") to 5 ("Disagree strongly"). All items were reverse-coded.

Finally, four individualism items were rated on 6-point scales ranging from 1 ("Very much like me") to 6 ("Not like me at all"): "It is important to think new ideas and being creative", "It is important to have a good time", "It is important to seek fun and do things that give pleasure", "It is important to make your own decisions and be free". All items were reverse-coded. Principal Component Analyses (PCA) were conducted to compute a single component for every cultural dimension, with a mean and standard deviation of 0 and 1 , respectively. For all scales, PCA corroborated the one-dimensional nature of the construct (explained variance and factor loading range for each dimension are displayed in Table $\mathrm{C}$ in SOM). Regional indicators were obtained by calculating the mean of individual factor scores on each dimension within a specific NUTS-region.

Anti-gay prejudice. Respondents rated three items on 5-point scales anchored by 1 ("Agree strongly") and 5 ("Disagree strongly"). The items read "Gays and lesbians are free to live life as they wish", "I would be ashamed if a close family member was gay or lesbian", 
and "Gay and lesbian couples should have the right to adopt children". After reverse-coding item 2, all items yielded a scale with $\alpha=.80 ; M=2.44(S D=1.13)$.

Sexist attitudes. For sexism, respondents answered the item "Men should have more right to job than women when jobs are scarce" on a scale ranging from 1 ("Agree strongly") to 5 ("Disagree strongly"). We reverse-coded this item $(M=1.96 ; S D=1.10)$. Both outcomes were positively interrelated $(r=.41, p<.001)$, and both positively correlated with religiosity ( $r=.25, p<.001$; and $r=.14, p<.001$, for anti-gay prejudice and sexism, respectively).

\section{Results}

The ICC was 0.36 for anti-gay prejudice and 0.16 for sexism. Tables 1 and 2 summarize all results. As in the previous studies, being religious and scoring higher on religiosity was related to higher prejudice levels, as were higher power distance, higher uncertainty avoidance, higher masculinity, and lower individualism. Again, a series of crosslevel interactions emerged. Multilevel simple slope analyses showed that stronger associations of religion and religiosity with anti-gay prejudice and sexism are found in regions low in power distance, uncertainty avoidance, masculinity and collectivism, whereas such associations are weaker in regions that score high on those cultural dimensions. Finally, to rule out statistical artefacts such as ceiling effects (which would be an alternative explanation for these results to the TCM), we calculated the value ranges in all "extreme" contexts (i.e., 2 SD above or below the mean). These data were not indicative of any ceiling or floor effects (see Table D in SOM).

\section{Discussion}

Our findings reveal a clear and consistent pattern: there are strong associations between religion and prejudice in regions low in power distance, uncertainty avoidance, masculinity and collectivism. Critically, the religion-prejudice link is constrained (i.e., weaker 
and often absent) in regions high on those cultural dimensions. The same pattern occurred across three large, representative samples, covering countries in the Americas and around the world (distal level) as well as European regions (proximal level). As predicted by the TCM, cultures that promote unequal/hierarchical relations (high power distance), strict rules (high uncertainty avoidance), "masculine" values (high masculinity), and group cohesion (high collectivism) tend to reflect an exclusionary, threatening climate. Not surprisingly, countries that rank highest on the threatening ends of each Hofstede dimension (e.g., Colombia, Ecuador, Guatemala, Iraq, Mexico, Russia, Venezuela, and Ukraine), also tend to rank high on objective threat indicators such as national homicide rate, unemployment, and economic inflation (see Onraet et al., 2013). The threatening climates in such countries tend to inhibit individual tendencies, and tend to mobilize almost everyone (also the non-religious) towards more homophobia and sexism (cf., Van Assche et al., 2017).

\section{Constraints to the Threat-Constraint Model}

That said, there are some boundaries to this conclusion. First, power distance and collectivism appear to be stronger constraints of religion-prejudice relationships than uncertainty avoidance and masculinity. Following Van Assche and colleagues (2017), our findings corroborate that cultures supporting economic-hierarchical group relations and ingroup unity tend to set a norm that appeals particularly those least prone to prejudice, while cultures emphasizing strict rules and masculine values do so to a lesser extent. Second, the mobilizing effect of proximal, regional cultures seems more potent than that of more distal, nationwide cultures. This is in line with the 'level of analysis' hypothesis stating that proximal geographical environments are most influential for social norms to have top-down effects on attitudes, since people spend most of their social life in their local area (Rentfrow, Gosling, \& Potter, 2008). 
Apart from the cross-sectional nature of the data, another limitation might be the suboptimal measurement of some constructs, which is a disadvantage inherent to using large worldwide datasets. For example, although we found threat-constraint effects for both religious affiliation and religiosity, the rather general operationalization of religiosity fails to capture its multifaceted nature. Whitley (2009), for instance, showed that greater intrinsic religious orientation was related to more homophobia, while greater extrinsic orientation was not. Hence, future studies could examine whether different components of religiosity all relate to gender- and sexual orientation-related attitudes in the same way. Moreover, it remains relatively unknown if similar person $\times$ context interactions would occur for all types of bigotry. These patterns were replicated for anti-gay prejudice and sexist attitudes, but individual-level religiosity and context-level cultural dimensions might not relate to racism or ageism in the same way. A final limitation in the current set of studies is that, due to lack of availability, the 'new' Hofstede dimensions (i.e., long-term orientation and indulgence) were not included, although they could also act as indicators of constraining contexts.

To conclude, this examination extends previous work (e.g., Hoffarth et al., 2018) by showing the key moderating role of Hofstede's cultural dimensions. On the 'threatening' end of each continuum, these dimensions have the power to eclipse the influence of individuals' own psychological makeup. Put differently, people's attitudes within a threatening culture tend to converge towards higher prejudice levels, regardless of their personal religious commitment. A major societal implication here is that, worldwide, such exclusionary climates may become normative — steering people not otherwise prone to endorse prejudice into outgroup derogation. Policy makers should consider this possibility when making decisions that may shape the perception of threat in our future societies. 


\section{References}

Adamczyk, A., \& Pitt, C. (2009). Shaping attitudes about homosexuality: The role of religion and cultural context. Social Science Research, 38(2), 338-351.

Allport, G. W. (1966). The religious context of prejudice. Journal for the Scientific Study of Religion, 5(3), 447-457.

Americas Barometer (2004-2014). Latin American Public Opinion Project (LAPOP), www.LapopSurveys.org.

De keersmaecker, J., Roets, A., Dhont, K., Van Assche, J., Onraet, E., \& Van Hiel, A. (2017). Need for closure and perceived threat as bases of right-wing authoritarianism: A longitudinal moderation approach. Social Cognition, 35(4), 433-449.

ESS Round 8: European Social Survey Round 8 Data (2016). Data file edition 2.1. Norwegian social science data services, Norway - Data archive and distributor of ESS data.

Glick, P. (2006). Ambivalent sexism, power distance, and gender inequality across cultures. In S. Guimond (Ed.), Social comparison and social psychology: Understanding cognition, intergroup relations, and culture (pp. 283-302). New York, NY, US: Cambridge University Press.

Glick, P., Lameiras, M., \& Castro, Y. R. (2002). Education and Catholic religiosity as predictors of hostile and benevolent sexism toward women and men. Sex Roles, 47(910), 433-441.

Hall, D. L., Matz, D. C., \& Wood, W. (2010). Why don't we practice what we preach? A meta-analytic review of religious racism. Personality and Social Psychology Review, 14(1), 126-139. 
Hoffarth, M. R., Hodson, G., \& Molnar, D. S. (2018). When and why is religious attendance associated with antigay bias and gay rights opposition? A justification-suppression model approach. Journal of Personality and Social Psychology, 115(3), 526.

Hofstede, G. (1980). Culture's consequences: International differences in work related values. Beverly Hills: Sage Publications.

Hox, J. J. (2013). Multilevel regression and multilevel structural equation modeling. The Oxford handbook of quantitative methods, 2(1), 281-294.

Kaasa, A., Vadi, M., \& Varblane, U. (2014). Regional cultural differences within European countries: evidence from multi-country surveys. Management International Review, 54(6), 825-852.

Leong, C. H., \& Ward, C. (2006). Cultural values and attitudes toward immigrants and multiculturalism: The case of the Eurobarometer survey on racism and xenophobia. International Journal of Intercultural Relations, 30(6), 799-810.

Navarro, M. C., Barrientos, J., Gómez, F., \& Bahamondes, J. (2019). Tolerance of homosexuality in South American countries: A multilevel analysis of related individual and sociocultural factors. International Journal of Sexual Health, 1-12.

Onraet, E., Van Hiel, A., \& Cornelis, I. (2013). Threat and right-wing attitudes: A crossnational approach. Political Psychology, 34(5), 791-803.

Raudenbush, S. W., \& Bryk, A. S. (2002). Hierarchical linear models: Applications and data analysis methods (2nd ed.). Thousand Oaks, CA: Sage. 
Rentfrow, P. J., Gosling, S. D., \& Potter, J. (2008). A theory of the emergence, persistence, and expression of geographic variation in psychological characteristics. Perspectives on Psychological Science, 3(5), 339-369.

Sibley, C. G., Osborne, D., \& Duckitt, J. (2012). Personality and political orientation: Metaanalysis and test of a Threat-Constraint Model. Journal of Research in Personality, 46(6), 664-677.

Triandis, H. C. (1995). Individualism and collectivism. Bouder, CO: Westview Press.

Van Assche, J., Koç, Y., \& Roets, A. (2019). Religiosity or ideology? On the individual differences predictors of sexism. Personality and Individual Differences, 139, 191197.

Van Assche, J., Roets, A., De keersmaecker, J. \& Van Hiel, A. (2017). The mobilizing effect of right-wing ideological climates: Cross-level interaction effects on different types of outgroup attitudes. Political Psychology, 38(5), 757-776.

Welzel, C., \& Inglehart, R. F. (2016). Misconceptions of measurement equivalence: Time for a paradigm shift. Comparative Political Studies, 49(8), 1068-1094.

Whitley Jr, B. E. (2009). Religiosity and attitudes toward lesbians and gay men: A metaanalysis. International Journal for the Psychology of Religion, 19(1), 21-38.

WVS Wave 6: World Values Survey Wave 6 (2010-2014). Official Aggregate v.20141107. World Values Survey Association, Aggregate File Producer: Asep/JDS, Madrid, Spain. 